\title{
Klebsiella rhinoscleromatis
}

National Cancer Institute

\section{Source}

National Cancer Institute. Klebsiella rhinoscleromatis. NCI Thesaurus. Code C86458.

A species of facultatively anaerobic, Gram negative, rod shaped bacteria assigned to the phylum Proteobacteria. This species is nonmotile and oxidase negative. K.

rhinoscleromatis is pathogenic and the causative agent of rhinoscleroma, an upper respiratory disease of the nose and pharynx. 